\title{
A Conceptual And Mechanistic Approach to UNDERSTANDING INTERACTIONS AMONG MULTIPLE Disturbance Agents: Compound Effects of Fire ON RESOURCE AVAILABILITY TO BARK BEETLES
}

\begin{abstract}
-
$\uparrow \quad$ INTRODUCTION

Population eruptions by native bark beetles are intermittent and can cause wide spread forest disturbance. The mountain pine beetle (MPB), Dendroctonus ponderosae Hopkins, is one such bark beetle currently affecting all species of pine in the Greater Yellowstone Ecosystem (GYE). MPB populations erupt after generating positive feedback once thresholds are surpassed. Population eruptions require a combination of factors such as favorable weather, tree susceptibility, and the reduced competitors and predators.
\end{abstract}

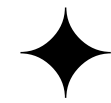

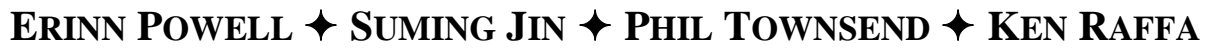 UNIVERSITY OF WISCONSIN $\uparrow$ MADISON}

Fire is also a major agent of disturbance throughout the GYE. Not all affected trees are killed, as some are only injured. Fire-injured trees may provide MPB with a suitably weakened host such that they could build-up their populations and reach eruptive population levels, subjecting healthy, vigorous trees to MPB caused mortality.

By conducting field work in Grand Teton National Park, Yellowstone National Park, and Bridger-Teton National Forest and working out of the UW-NPS Research Station, we are currently investigating the following question:

Does fire injury in lodgepole pine affect colonization rates, reproductive success, and potential for population increase of mountain pine beetle?
Objective \#1: Mountain pine beetle colonization of fire-injured lodgepole pine

Four sites in Yellowstone National Park, Grand Teton National Park, and Bridger-Teton National Forest that burned during summer 2006 were studied in the summer of 2007 , and four sites that burned during summer 2007 were studied in the summer of 2008 (Figure 1). Four $100 \mathrm{~m}$ x $5 \mathrm{~m}$ belt transects at each site were used to determine the number of lodgepole pines per fire injury category (none to low, moderate, high), the presence or absence of the mountain pine beetle in each tree, and the presence or absence of mountain pine beetle competitors. Our results indicate that fire injury predisposes trees to mountain pine beetle colonization, but the extent to which this occurs varies in a nonlinear fashion with the severity of injury (Chi Square analysis, $p<0.001$ ). Analysis is ongoing, and the project will be completed in 2010.

Objective \#2: Mountain pine beetle reproductive success and interspecific competition within fireinjured lodgepole pine

A subsample of 30 lodgepole pines of each fire-injure category (as described in Objective \#1) were designated for sampling in 2007 and 2008. The holes of entry and exit for the mountain pine beetles from breast height to forest floor will be marked and counted for each pine. The larval galleries for the mountain pine beetle and mountain pine beetle competitors will be measured. Data collection and analysis will continue through 2010. 


\section{Objective \#3: Potential mountain pine beetle population increase in burned lodgepole pine}

The relationship between tree mortality due to mountain pine beetle and proximity to burn perimeter is currently being tested. To control for regional context, we will test different measures of beetle population dynamics derived from the remote sensing analysis and insect pheromone trapping at all 8 burn sites as covariates in the logistic models. Analyses are underway, and the project will continue through 2010.

\section{Objective \#4: Host resistance and suitability}

The resistance of fire-injured lodgepole pine to bark beetles was determined by measuring the amount of resin and the concentration and composition of monoterpenes present in constitutive and induced phloem from 50 lodgepole pines of each

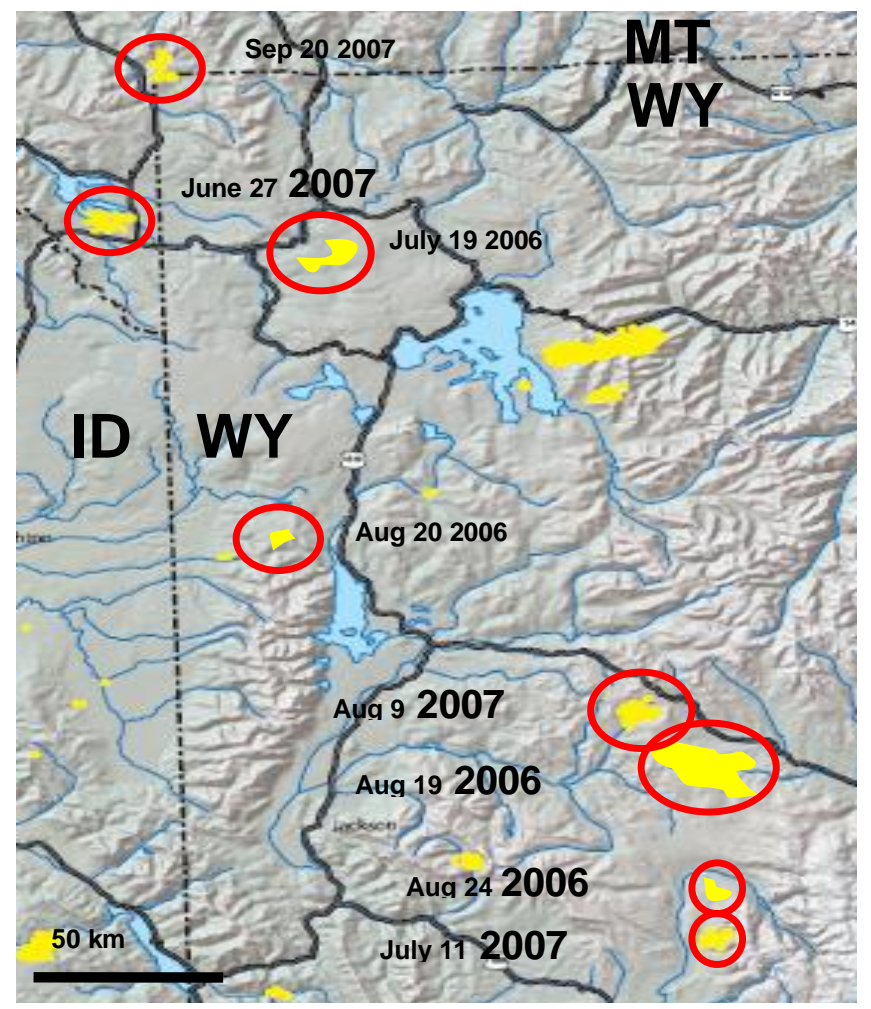

Figure 1. Eight burns throughout the Greater Yellowstone Ecosystem. All were sampled in 2007 and 2008. Dates indicate the date of burn. fire injury category (as described in Objective \#1). Resin flow was measured by puncturing the bark of each pine and attaching a graduated centrifuge tube to the wound site. Resin flow was found to differ across the categories (Kruskal-Wallis $p=2.674 \times 10^{-}$ $\left.{ }^{9}\right)$. Phloem $\left(3 \mathrm{inch}^{2}\right)$ was removed from each pine, immediate frozen, and shipped to Wisconsin for monoterpene analysis. Monoterpenes are currently being analyzed by gas chromatography.

The suitability of fire-injured lodgepole pine to bark beetles was determined by identifying the fungal composition, and the concentration of nitrogen in the phloem of the 50 lodgepole pines of each fire injury category. Phloem (2cm diameter) was removed from each pine and plated onto malt extract agar. The acquired fungus was allowed to grow on each plate. Identification of the fungi is ongoing. Phloem $\left(1 \mathrm{inch}^{2}\right)$ was removed from the pines and dried to determine the concentration of nitrogen. This analysis is also ongoing. 